\title{
Efeito de quatro tipos de mel na longevidade e reprodução de Catolaccus grandis (Hymenoptera:Pteromalidae).
}

\author{
Effect of four types of honey on longevity and reproduction of Catolaccus grandis \\ (Hymenoptera:Pteromalidae)
}

\author{
Paulo Alves Wanderley ${ }^{1}$ Maria José Araújo Wanderley ${ }^{2}$ \\ Arlindo Leal Boiça Júnior ${ }^{3}$ Álvaro Pavan Júnior ${ }^{4}$
}

\section{RESUMO}

O trabalho teve como objetivo conhecer o melhor tipo de mel em relação à longevidade e fertilidade do parasitóide do bicudo-do-algodoeiro Catolaccus grandis. Adultos recém-emergidos foram transferidos para recipientes plásticos de $500 \mathrm{~mL}$, adaptados com tubo para fornecer água e umidade para os insetos. Um casal do parasitóide foi mantido em cada recipiente em câmara climatizada a $25 \pm 1{ }^{\circ} \mathrm{C}$, $U R=70 \pm 10 \%$ e fotoperíodo de 14h. O trabalho constou de 4 tratamentos: mel de flor de laranjeira; mel de flores de plantas silvestres; mel de cana-de-açúcar (todos produzidos por Apis mellifera) e mel de flores silvestres produzido por abelha Jataí (Tetragonistica angustula), com 15 repetições cada. Adultos recém-emergidos do parasitóide receberam cinco larvas de Euscepes postfasciatus encapsuladas em parafilm. Três gotículas de mel foram colocadas sobre o parafilm. Calculou-se a longevidade de machos e fêmeas, número de ovos dia ${ }^{-1}$ fêmea ${ }^{-1}$ e número total de ovos fêmea ${ }^{-1} e$ construíram-se as tabelas de fertilidade. As melhores dietas para alimentar adultos de $\boldsymbol{C}$. grandis foram o mel silvestre $e$ $o$ de laranjeira. A melhor fecundidade foi observada na dieta de mel de laranjeira (101,60 ovos fêmea $\left.{ }^{-1}\right)$ e os melhores resultados para aumento reprodutivo e populacional deste parasitóide foram obtidos com mel de laranja e com mel silvestre.

Palavras-chave: Catolaccus grandis, mel, tabela de vida, longevidade, fertilidade.

\section{ABSTRACT}

The aim of this work was to investigate the best type of honey in relation to longevity and life fertility of Catolaccus grandis. Recent emerged adults were transferred to $500 \mathrm{ml}$ plastic recipients adapted to supply water and moisture to insects. A couple of parasite was kept for each recipient in climatic chamber at $25 \pm 1{ }^{\circ} \mathrm{C}, \mathrm{RH}=70 \pm 10 \%$ and photofase $14 \mathrm{~h}$. The work had four treatments: orange, wild plant species honey, Jatai
Tetragonistica angustula and sugar cane honey with fifteen replications each. Recent emerged adults received five Euscepes postfasciatus larvae, which were encapsulated in parafilm. Three drops of honey from respective treatment were placed on parafilm. Longevity of males and females were calculated, as well as the number of eggs female ${ }^{-1}$. Tables of fertility were made. The best diets to feed adults of $\boldsymbol{C}$. grandis were wild plant species honey and orange honey; the best fecundity was observed on orange honey diet (101,60 eggs female $\left.{ }^{-1}\right)$ and the parameters of life fertility table were obtained using diets based on orange honey and wild plant species honey.

Key words: Catolaccus grandis, honey, life table, longevity, fertility.

\section{INTRODUÇÃO}

No Brasil, o algodoeiro (Gossypium hirsutum) é uma cultura de tradicional importância econômica e social, tanto na produção de fibra quanto na indústria alimentícia (NEVES \& JUNQUEIRA, 1965). Pesquisadores têm chamado a atenção sobre a importância econômica e ecológica do uso de parasitóides, como estratégia para reduzir populações do bicudo no Brasil. Esses artrópodes contribuem para o controle do bicudo, nos diferentes agroecossistemas do algodoeiro, alimentando-se interna ou externamente das formas imaturas de $\boldsymbol{A}$. grandis (PIEROZZI JÚNIOR, 1985). No Brasil, RAMALHO \& WANDERLEY (1996) registraram a ocorrência de 13 espécies de parasitóides que atacam o bicudo, sendo que na Região Nordeste a mortalidade natural do bicudo, causada por parasitismo, deve-se, principalmente, à ação dos

\footnotetext{
${ }^{1}$ Professor, Doutor, Universidade Federal da Paraíba (UFPB), Centro de Formação de Tecnólogo (CFT). Rua Antônio Alves da Rocha, 12, conj. Edgar St ${ }^{a}$ Cruz, 58220-000, Bananeiras, PB. E-mail: alwanderley@iwpb.com.br.

${ }^{2}$ Doutora em Agronomia, Bolsista de Desenvolvimento Regional, Conselho Nacional de Desenvolvimento Científico e Tecnológico $(\mathrm{CNPq}) / \mathrm{UFPB}$.

${ }^{3}$ Professor, Doutor, em Agronomia, Universidade Estadual Paulista (UNESP), Faculdade de Ciências Agrárias e Veterinárias (FCAV), Bolsista de Pesquisa, CNPq.

${ }^{4}$ Engenheiro Agrônomo, UNESP-Jaboticabal.
} 
parasitóides Catolaccus grandis (Hymenoptera: Pteromalidae) e Bracon vulgaris Ashmead (Hymenoptera: Braconidae) (RAMALHO, 1994). Adultos de $\boldsymbol{C}$. grandis foram observados por ADAMS et al. (1969) alimentando-se de néctar, começando pelos nectários de botões florais, flores e folhas da própria planta de onde emergiram. Pesquisas também têm demonstrado que o uso de hospedeiros alternativos para a criação de $\boldsymbol{C}$. grandis é comercialmente viável. ROJAS et al. (1998) observaram que larvas de Callosobruchus maculatus (Coleoptera: Bruchidae) é um hospedeiro ideal para a criação de $\boldsymbol{C}$. grandis. Considerando-se que $\boldsymbol{C}$. grandis é um parasitóide do bicudo-do-algodoeiro, introduzido no Brasil, torna-se imprescindível que se desenvolvam estudos relacionando dietas que proporcionem maiores longevidade e fertilidade para fêmeas desse parasitóide. O objetivo da pesquisa foi gerar informações sobre o tipo de mel mais adequado para a alimentação de $\boldsymbol{C}$. grandis, a fim de proporcionar maiores longevidade e fertilidade.

\section{MATERIAL E MÉTODOS}

A pesquisa foi realizada no Laboratório de Resistência de Plantas a Insetos, Departamento de Fitossanidade, FCAV/UNESP, Jaboticabal, SP. Os hospedeiros alternativos utilizados para a criação do parasitóide foram larvas e pupas da broca $(\boldsymbol{E}$. postfasciatus) da batata-doce (Ipomoea batatas). A criação desse hospedeiro foi iniciada com adultos fornecidos pela Unidade de Controle Biológico/ EMBRAPA - Algodão, Campina Grande, PB.

Baldes de 10L contendo quatro a cinco batatas doce foram infestados com adultos da broca, na proporção de 15 brocas para cada batata. Esses baldes foram vedados na parte superior com um tecido de cor preta, proporcionando um ambiente adequado para a sobrevivência e reprodução das brocas. Após uma semana da infestação, as batatas foram retiradas e transferidas, sem as brocas, para um outro recipiente como descrito acima, visando à eclosão das larvas de E. postfasciatus. As larvas e pupas presentes nessas batatas, com cerca de um mês, foram utilizadas na pesquisa com os parasitóides.

O trabalho foi desenvolvido com adultos de $\boldsymbol{C}$. grandis recém emergidos, mantidos em recipientes plásticos transparentes de $500 \mathrm{~mL}$, com orifícios na extremidade superior nos quais foram inseridos tubos recicláveis de anestésicos odontológicos, contendo água e vedados com algodão, para o fornecimento de água e umidade. Em cada recipiente, foi distribuído um casal do parasitóide C. grandis que foi mantido em câmara climatizada a $25^{\circ} \mathrm{C} \pm 1$ e umidade relativa de $70 \pm 10 \%$. Utilizaram-se quatro tratamentos com 15 repetições cada: 1 . mel de flor de laranjeira; 2 . mel de flores de plantas silvestres; 3. mel de cana-de-açúcar, todos produzidos por Apis mellifera florada de Jaboticabal-SP, 2001 e 4. mel de flores silvestres produzido por abelha Jataí ( $\boldsymbol{T}$. angustula) também da florada de Jaboticabal em 2001. Após três dias da emergência desses parasitóides foram oferecidas, diariamente, 5 larvas e/ou pupas de E. postfasciatus encapsuladas em Parafilm para oviposição por parte dos parasitóides. Distribuíramse três gotículas de mel, diluído em água a uma proporção de 1:10, entre as células formadas com parafilme, de acordo com o tratamento. A metodologia de criação e manutenção de $\boldsymbol{C}$. grandis foi conforme a descrita por CATE (1987) apresentando algumas modificações sugeridas por RAMALHO \& WANDERLEY (1996).

As celas com o hospedeiro parasitado foram transferidas para copos descartáveis e mantidas em câmaras climatizadas sob as mesmas condições em que se encontravam os genitores, a fim de que ocorresse a eclosão das larvas de $\boldsymbol{C}$. grandis. As pupas do parasitóide foram separadas de acordo com o sexo. Cada casal de $\boldsymbol{C}$. grandis foi observado diariamente, avaliando-se a longevidade de fêmeas e machos, número médio diário e total de ovos por cada fêmea do parasitóide sobre cada larva de $\boldsymbol{E}$. postfasciatus. Utilizou-se o delineamento inteiramente casualizado. Esses dados foram submetidos à análise de variância e as médias comparadas pelo teste de Tukey. Com os dados de sobrevivência, número de postura por fêmea, número de ovos por postura por fêmea e número de ovos por fêmea foram construídas tabelas de fertilidade para os adultos de $\boldsymbol{C}$. grandis, conforme SILVEIRA NETO et al. (1976). De posse dos dados de fertilidade, foi calculada a razão infinitesimal de aumento em número de uma população $\left(\mathrm{r}_{\mathrm{m}}\right)$, aplicando-se a equação de CAREY (1993) $r_{m}=\ln R_{0} / T$; onde $R_{0}$ representa a taxa líquida de reprodução, ou seja, o número médio de fêmeas produzidas por uma única fêmea do parasitóide durante sua vida média (KREBS, 1994). A razão finita de aumento da população $(\lambda)$ foi calculada através da fórmula $\lambda=$ anti log. $\left(r_{m} .0,4343\right)$ (KREBS, 1994) que consiste no número de indivíduos adicionados à população por fêmea do predador por dia. A duração de uma geração (T), que corresponde ao período médio que vai do nascimento dos genitores ao nascimento dos descendentes, foi calculada pela fórmula $\mathrm{T}=\Sigma \mathrm{m}_{\mathrm{x}} \cdot \mathrm{l}_{\mathrm{x}} \cdot \mathrm{x} / \Sigma \mathrm{m}_{\mathrm{x}} \cdot \mathrm{l}_{\mathrm{x}}$. 


\section{RESULTADOS E DISCUSSÃO}

Os diferentes tipos de mel utilizados na alimentação de adultos de $\boldsymbol{C}$. grandis influenciaram a longevidade média (Tabela 1). Observou-se que quando se ofereceu dieta composta de mel silvestre e de laranjeira, machos e fêmeas apresentaram longevidade mais alta, com valores médios de $33,33 \mathrm{e}$ 29,33 dias (machos) e de 33,87 e 29,33 dias (fêmeas), respectivamente. Quando alimentados com mel de Jataí e de cana-de-açúcar a longevidade foi menor, com médias de 14,20 dias e 11,87 dias (machos) e de 11,27 dias e 10,93 dias (fêmeas), respectivamente, provavelmente pelo maior teor de açúcares simples e de mais fácil digestão pelos parasitóides existentes no mel de laranja e mel silvestre quando comparados com os outros tipos estudados. Por outro lado, MORALES-RAMOS \& CATE (1992) observaram longevidade da fêmea de $\boldsymbol{C}$. grandis de 63,6 \pm 15,12 dias, criadas a temperatura de $30 \pm 1^{\circ} \mathrm{C}$ e alimentadas com solução de mel de abelha (origem não especificada) e água destilada. Caso se almeje um ciclo de vida mais longo dos indivíduos, deve-se utilizar mel silvestre ou mel de laranjeira na alimentação de $\boldsymbol{C}$. grandis tendo como hospedeiro larvas de $\boldsymbol{E}$. postfasciatus.

Quando se utilizaram mel silvestre e mel de laranjeira na alimentação de $\boldsymbol{C}$. grandis, observou-se a maior média do número de ovos por fêmea por dia $(2,93$ e 5,00), respectivamente (Tabela 1$)$. Da mesma forma, o número total de ovos por fêmea foi maior $(101,60)$ quando se utilizou mel de laranjeira. O número de ovos por fêmea por dia, bem como o número total de ovos por fêmea foi menor quando alimentados com mel de cana-de-açúcar. A biologia de um parasitóide pode ser afetada de acordo com a alimentação (PRICE, 1986). Assim, visando uma maior produção de

Tabela 1 - Longevidade média, número de ovos fêmea ${ }^{-1}$ dia $^{-1}$ e número de ovos fêmea $^{-1}$ de Catolaccus grandis, sob diferentes dietas.

\begin{tabular}{lllll}
\hline & \multicolumn{2}{l}{ Longevidade } & Número de ovos \\
\cline { 2 - 5 } Dietas & Machos & Fêmeas & Diário & Total \\
\hline Mel silvestre & $33,33 \mathrm{a}^{1}$ & $33,87 \mathrm{a}$ & $2,93 \mathrm{a}$ & $48,10 \mathrm{~b}$ \\
Mel laranjeira & $29,33 \mathrm{a}$ & $29,33 \mathrm{a}$ & $5,00 \mathrm{a}$ & $101,60 \mathrm{a}$ \\
Mel Jataí & $14,20 \mathrm{~b}$ & $11,27 \mathrm{~b}$ & $1,73 \mathrm{bc}$ & $6,50 \mathrm{c}$ \\
Mel cana & $11,87 \mathrm{~b}$ & $10,93 \mathrm{~b}$ & $0,91 \mathrm{c}$ & $3,60 \mathrm{c}$ \\
Média total & $22,18 \pm 5,37$ & $21,35 \pm 5,99$ & $2,64 \pm 0,89$ & $39,95 \pm 22,93$ \\
CV $(\%)$ & 24,61 & 25,08 & 41,08 & 76,25 \\
\hline
\end{tabular}

${ }^{1}$ Médias seguidas de letras diferentes nas colunas diferem estatisticamente pelo teste de Tukey, em nível de $1 \%$ de probabilidade. indivíduos quando submetidos a uma dessas dietas, deverá se utilizar o mel de laranjeira na alimentação de C. grandis. O mel de laranjeira é produzido pelas abelhas melíferas a partir do néctar extraído de nectários florais formados estrategicamente para atrair, especialmente insetos, para a polinização (COUTO \& COUTO, 2002), contendo componentes nutricionais propícios ao desenvolvimento dos insetos.

Dados da fertilidade de $\boldsymbol{C}$. grandis (Tabela 2) mostraram que as fêmeas apresentaram-se aptas a produzirem fêmeas $\left(\mathrm{m}_{\mathrm{x}}\right)$ a partir de $8,10,10$ e 13 dias, respectivamente, quando alimentadas com as dietas de mel de laranjeira, mel silvestre, mel de Jataí e mel de cana-de-açúcar. Alguns parasitóides, ao emergirem, não estão prontos para a reprodução, necessitando para isso, ingerir alimentos ricos em açúcares e aminoácidos (GARCIA, 1991). O registro de dados possibilita fazer estimativas do número de fêmeas esperadas que serão produzidas por fêmea de idade $\mathrm{x}$, viva naquela idade (PRICE, 1975). Assim, o maior pique de produção de fêmeas por fêmea ocorreu quando $\boldsymbol{C}$. grandis foi alimentado com mel de laranjeira (Tabela 2), resultando num total de 7,33 fêmeas por fêmeas adultas com 28 dias de idade, decrescendo essa produção a partir daí até o $48^{\circ}$ dia.

O suprimento alimentar do parasitóide com mel silvestre proporcionou uma maior produção de fêmeas por fêmea entre o intervalo de idade de 30 $(2,45)$ e 35 (2,34 fêmeas) dias (Tabela 2). Quando alimentadas com mel de Jataí, a produção de fêmeas por fêmea foi maior $(1,120$ fêmeas) quando o parasitóide se encontrava na classe de idade 15 (Tabela 2), o que significa que, nessa idade, as fêmeas atingiram o máximo de sua capacidade de produção de ovos.

Fêmeas alimentadas com mel de cana-deaçúcar apresentaram valores de $\mathrm{m}_{\mathrm{x}}$ menores que 1

(Tabela 2), indicando que a produção de fêmeas quando alimentadas com essa dieta será muito baixa. A importância da produção de fêmeas consiste no fato de que são esses insetos que irão atacar o hospedeiro, tanto para paralisá-lo como para ovipositar. Os insetos que foram alimentados com mel de laranjeira e mel silvestre viveram mais (48 dias e 45 dias, respectivamente), do que quando alimentados com mel de Jataí (29 dias) e de cana-de-açúcar (26 dias) (Tabela 2).

$O$ valor de $R_{0}$, que mostra a capacidade de aumento da população de C. grandis, foi de 21,$58 ; 7,47 ; 0,35$ e 0,28 descendentes fêmeas que deram origem 
Tabela 2 - Fertilidade de vida para Catolaccus grandis alimentados com diferentes dietas tendo como hospedeiro larvas de Euscepes postfasciatus.

\begin{tabular}{|c|c|c|c|c|c|}
\hline Dieta & $x^{1}$ & $\mathrm{~m}_{\mathrm{x}}$ & $1_{\mathrm{x}}$ & $\mathrm{m}_{\mathrm{x}} \cdot 1_{\mathrm{x}}$ & $\mathrm{m}_{\mathrm{x}} \cdot 1_{\mathrm{x}} \cdot \mathrm{x}$ \\
\hline \multirow[t]{10}{*}{ Mel de laranjeira } & 03 & 0,000 & 0,000 & 0,000 & 0,000 \\
\hline & 08 & 0,190 & 1,000 & 0,190 & 1,520 \\
\hline & 13 & 3,120 & 1,000 & 3,120 & 40,520 \\
\hline & 18 & 4,310 & 1,000 & 4,310 & 77,580 \\
\hline & 23 & 4,540 & 1,000 & 4,540 & 104,420 \\
\hline & 28 & 7,330 & 0,867 & 6,360 & 177,940 \\
\hline & 33 & 6,760 & 0,400 & 2,700 & 89,230 \\
\hline & 38 & 0,860 & 0,330 & 0,280 & 10,780 \\
\hline & 43 & 1,050 & 0,067 & 0,070 & 3,030 \\
\hline & 48 & 0,000 & 0,000 & 0,000 & 0,000 \\
\hline \multirow[t]{9}{*}{ Mel silvestre } & 05 & 0,000 & 0,000 & 0,000 & 0,000 \\
\hline & 10 & 0,329 & 1,000 & 0,329 & 3,290 \\
\hline & 15 & 1,130 & 1,000 & 1,130 & 16,950 \\
\hline & 20 & 0,710 & 1,000 & 0,710 & 14,200 \\
\hline & 25 & 1,860 & 1,000 & 1,860 & 46,500 \\
\hline & 30 & 2,450 & 0,867 & 2,120 & 63,720 \\
\hline & 35 & 2,340 & 0,467 & 1,090 & 38,250 \\
\hline & 40 & 1,650 & 0,133 & 0,220 & 8,780 \\
\hline & 45 & 0,000 & 0,000 & 0,000 & 0,000 \\
\hline \multirow[t]{6}{*}{ Mel de abelha Jataí } & 05 & 0,000 & 0,000 & 0,000 & 0,000 \\
\hline & 10 & 0,059 & 0,800 & 0,050 & 0,470 \\
\hline & 15 & 1,120 & 0,267 & 0,300 & 4,490 \\
\hline & 20 & 0,000 & 0,067 & 0,000 & 0,000 \\
\hline & 25 & 0,000 & 0,000 & 0,000 & 0,000 \\
\hline & 29 & 0,000 & 0,000 & 0,000 & 0,000 \\
\hline \multirow[t]{6}{*}{ Mel de cana-de-açúcar } & 03 & 0,000 & 0,000 & 0,000 & 0,000 \\
\hline & 08 & 0,000 & 0,000 & 0,000 & 0,000 \\
\hline & 13 & 0,233 & 0,690 & 0,160 & 2,090 \\
\hline & 18 & 0,530 & 0,230 & 0,120 & 2,190 \\
\hline & 23 & 0,000 & 0,077 & 0,000 & 0,000 \\
\hline & 26 & 0,000 & 0,000 & 0,000 & 0,000 \\
\hline
\end{tabular}

$\mathrm{x}=$ intervalo de idade (dia); $\mathrm{m}_{\mathrm{x}}=$ fêmeas produzidas por fêmea de idade $\mathrm{x} ; 1_{\mathrm{x}}=$ taxa de sobrevivência a partir da idade zero ao começo da idade $\mathrm{x} ; \mathrm{m}_{\mathrm{x}} \cdot \mathrm{l}_{\mathrm{x}}=$ expectativa de reprodução na idade $\mathrm{x}$.

a fêmeas reprodutivas no curso de uma geração, quando alimentadas com as dietas de mel de laranjeira, mel silvestre, mel de Jataí e mel de cana-de-açúcar, respectivamente, (Tabela 3 ), sugerindo que, provavelmente, as fêmeas adicionaram mais fêmeas à população quando alimentadas com as dietas de mel de laranjeira e silvestre. Sendo os valores de $R_{o}$ maiores quando as fêmeas foram alimentadas com essas duas dietas, provavelmente a taxa líquida de reprodução foi afetada pela dieta utilizada. Quando as fêmeas foram alimentadas com o mel de laranjeira e silvestre, os valores para a razão finita de aumento da população (l) tenderam a ser maiores, indicando que $\boldsymbol{C}$. grandis alimentado com essas dietas provavelmente adicionará mais fêmeas à população por fêmea em um mesmo intervalo de tempo, do que quando alimentado com as demais dietas, ou seja, adicionará 1,14 e 1,08 fêmeas à população por dia, respectivamente (Tabela 3 ).

O mel produzido por $\boldsymbol{A}$ melllifera possui grandes quantidades de açúcares simples (32\% de glicose e $38 \%$ de frutose), minerais como: potássio, sódio, cloro, enxofre, cálcio, fósforo, silício, ferro e magnésio, além de aminoácidos, enzimas e vitaminas (COUTO \& COUTO, 2002). O mel silvestre, também chamado de multifloral, tem sua composição muito variável dependendo de diversos fatores, como espécies com flores disponíveis e época do ano. $\mathrm{O}$ mel de cana, por sua vez, possui alto teor de sacarose que é digerido mais lentamente que o mel rico em glicose e frutose. Quanto ao mel de Jataí, as informações são raras. KERR et al. (1996) afirmam apenas que o mel dos meliponídeos, como é o caso de Jataí, possui até $70 \%$ de açúcares. Esses aspectos parecem afetar o desempenho reprodutivo das fêmeas de $\boldsymbol{C}$. grandis, uma vez que o mel de cana promoveu um desempenho inferior em relação aos demais.

Em outro estudo $\boldsymbol{C}$. grandis apresentou valores de $R_{o}$ de 11,97 e de $r_{m}$ de 0,066 (RAMALHO et al., 1998), resultados compatíveis com os encontrados neste trabalho, quando os parasitóides foram alimentados com mel silvestre. As durações médias de uma geração $(\mathrm{T}) \mathrm{de}$ C. grandis foram de 23,40; 25,66; 14,16 e 15,30 dias, respectivamente, quando as fêmeas foram alimentadas com o mel de laranjeira, silvestre, de Jataí e de cana-de-açúcar. Quando as fêmeas de $\boldsymbol{C}$. grandis foram alimentadas com mel de Jataí e mel de cana-deaçúcar, registraram-se razões infinitesimais de aumento $\left(r_{m}\right)$ negativas $(-0,07$ e $-0,08$, respectivamente), resultando em valores da razão finita de aumento menores que 1. Esses dados sugerem que, quando $\boldsymbol{C}$. grandis é alimentado com essas dietas, não ocorre crescimento populacional desse parasitóide.

\section{CONCLUSÃO}

As fêmeas de $\boldsymbol{C}$. grandis alimentadas com mel de laranjeira ou silvestre aumentam o potencial reprodutivo em relação àquelas alimentadas com mel de Jataí e de cana-de-açúcar. A dieta de mel de laranjeira proporciona melhores resultados para longevidade e fertilidade que as demais dietas, quando o hospedeiro do $\boldsymbol{C}$. grandis é $\boldsymbol{E}$. postfasciatus. 
Tabela 3 - Variáveis reprodutivas de Catolaccus grandis alimentados com diferentes dietas, tendo como hospedeiro larvas de Euscepes postfasciatus.

\begin{tabular}{lcrrr}
\hline Dietas & $\mathrm{T}^{1}$ & $\mathrm{R}_{\mathrm{o}}$ & $\mathrm{r}_{\mathrm{m}}$ & $\lambda$ \\
\hline Mel de laranjeira & 23,40 & 21,58 & 0,1313 & 1,14 \\
Mel silvestre & 25,66 & 7,47 & 0,0784 & 1,08 \\
Mel de Jataí & 14,16 & 0,35 & $-0,07$ & 0,93 \\
Mel de cana-de-açúcar & 15,30 & 0,28 & $-0,08$ & 0,92 \\
\hline
\end{tabular}

${ }^{1} \mathrm{~T}=$ duração média de uma geração (dia); $\mathrm{R}_{\mathrm{o}}=$ taxa líquida de reprodução; $\mathrm{r}_{\mathrm{m}}=$ razão infinitesimal de aumento.

\section{REFERÊNCIAS BIBLIOGRÁFICAS}

ADAMS, C.H.; CROSS, W.H.; MITCHELL, H.C. Biology of Bracon mellitor, a parasite of the boll weevil. Journal of Economic Entomology, Lanham, v.62, n.4, p.889-895, 1969.

CAREY, J.R. Applied demography for biologists with special emphasis on insects. New York : Oxford University, 1993. 206p.

CATE, J.R. A method of rearing parasitoids of boll weevil without the host plant. The Southwestern Entomologist, Texas, v.12, n.3, p.211-215, 1987 .

COUTO, R.H.N.; COUTO, L.A. Apicultura: manejo e produtos. Jaboticabal-SP : FUNEP, 2002. $191 \mathrm{p}$.

GARCIA, M.A. Ecologia nutricional de parasitóides e predadores terrestres. In: PANIZZI, A.R.; PARRA, J.R.P. Ecologia nutricional de insetos e suas implicações no manejo de pragas. São Paulo : Manole, 1991. Cap.8, p.289-311.

KERR, W.E.; CARVALHO, G.A.; NASCIMENTO, V.A. Abelha uruçú: biologia e manejo. Belo Horizonte : Acangaú, 1996. $144 \mathrm{p}$.

KREBS, C.J. Ecology: the experimental analysis of distribution and abundance. New York : Harper \& Row, 1994. 801p.
MORALES-RAMOS, J.A.; CATE, J.R. Rate of increase and adult longevity of Catolaccus grandis (Burks) (Hymenoptera: Pteromalidae) in the laboratory at four temperatures. Environmental entomology, Lanham, v.21, n.3, p.620-627, 1992.

NEVES, O.S.; JUNQUEIRA, A. A B. In: O algodão no Brasil: cultura e adubação do algodoeiro. São Paulo: INSTITUTO BRASILEIRO DE POTASSA, 1965. Cap. 2:. p.55-115.

PIEROZZI JUNIOR, I. Ecologia aplicada de Anthonomus grandis Boheman, 1843 (Coleoptera: Curculionidae) na região de Campinas. 1985. 155f. Dissertação (Mestrado em Ecologia) - Curso de Pós-graduação em Ecologia, Universidade Estadual de Campinas.

PRICE, P.W. Insect ecology. New York : John Wiley \& Sons, 1975. 514p.

PRICE, P.W. Ecological aspects of host plant resistance and biological control: interactions among three trophic levels. In: BOETHEL, D.J.; EIKENBARY, R.D. (Eds). Interactions of plant resistance and parasitoids and predators of insects. New York : Ellis Horwood, 1986. p.11-30.

RAMALHO, F.S. Cotton pest management: part.4. A brazilian perspective. Annual Review of Entomology, Stanford, v.39, p.553-578, 1994.

RAMALHO, F.S.; WANDERLEY, P.A. Ecology and management of cotton boll weevil in South American cotton. American entomologist, Lanham, v.42, n.1, p.41-47, 1996.

RAMALHO, F.S.; WANDERLEY, P.A.; MEZZOMO, J.A. Influência da temperatura na fecundidade e ataque de Catolaccus grandis (Burks) (Hymenoptera, Pteromalidae), parasitóide do bicudo-do-algodoeiro, Anthonomus grandis Boheman (Coleoptera, Curculionidae). Revista brasileira de entomologia, Curitiba, v.42, n.1/2, p.7178,1998

ROJAS, M.G. et al. Use of a factitious host and supplemented adult diet to rear and induce oogenesis in Catolaccus grandis (Hymenoptera: Pteromalidae). Environmental Entomology, Lanham, v.27, p.499507, 1998 .

SILVEIRA NETO, S. et al. Manual de ecologia dos insetos. São Paulo: Agronômica Ceres, 1976. $419 \mathrm{p}$. 